\title{
Germanica
}

\section{« kostbar ist uns nur der augenblick des erkennens »}

Une introduction à la lecture de Chlebnikov weint (2015) d'Anne Seidel

Laurent Cassagnau: "kostbar ist uns nur der augenblick des erkennens". Eine

Einführung in Anne Seidels Chlebnikov weint (2015)

Laurent Cassagnau: "kostbar ist uns nur der augenblick des erkennens“. An

introduction to Anne Seidel's Chlebnikov weint (2015)

\section{Laurent Cassagnau}

\section{(2penEdition}

\section{Journals}

Édition électronique

URL : http://journals.openedition.org/germanica/6798

DOI : 10.4000/germanica.6798

ISSN : 2107-0784

\section{Éditeur}

Université de Lille

\section{Édition imprimée}

Date de publication : 26 juin 2019

Pagination : 101-114

ISBN : 978-2-913857-42-1

ISSN : 0984-2632

\section{Référence électronique}

Laurent Cassagnau, « «kostbar ist uns nur der augenblick des erkennens » », Germanica [En ligne], 64 | 2e trimestre 2019, mis en ligne le 01 janvier 2021, consulté le 27 février 2021. URL : http:// journals.openedition.org/germanica/6798; DOI : https://doi.org/10.4000/germanica.6798 


\title{
« kostbar ist uns nur der augenblick des erkennens $\gg$.
}

\author{
Une introduction à la lecture de Chlebnikov \\ weint (2015) d'Anne Seidel
}

\author{
Laurent Cassagnau \\ ENS, Lyon
}

Dans son recueil Tristia (1916), inspiré des élégies d'Ovide et de Tibulle, le poète russe Ossip Mandelstam écrit : " Tout a été, et ce qui fut sera, / Seule est douce l'heure où tu reconnais. $\rangle^{1}$. Cette citation est reprise et développée en libre traduction allemande dans Chlebnikov weint $^{2}$, premier recueil de la poétesse allemande Anne Seidel ${ }^{3}$ paru en 2015, qui comporte de nombreuses références à la littérature et à la culture russes, et plus généralement slaves. Dans le poème intitulé

1. - Ossip Mandelstam, Euvres complètes I (Euvres poétiques), édition bilingue, trad. Jean-Claude Schneider, Paris, Le Bruit du temps/La Dogana, 2018. Mandelstam reprend ce vers de Tristia dans son essai « Verbe et culture » (ibid., p. 326).

2. - Anne Seidel, Chlebnikov weint. Gedichte, hrsg. v. Jane-Ann Igel, Jan Kuhlbrodt, Ralph Lindner, Reihe Neue Lyrik der Kulturstiftung des Freistaates Sachsen, Bd. 9., Leipzig, Poetenladen Verlag, 2015, p. 12-13 (abrégé par la suite en $C W$ ).

3. - Anne Seidel est née en 1988 à Dresde. Elle a fait des études de slavistique et d'histoire de l'Europe orientale à l'université de Giessen. Elle a par ailleurs initié divers projets d'art sonore. Chlebnikov weint a été recommandé par la Deutsche Akademie für Sprache und Dichtung et a été distingué en 2016 par le Literaturwerkstatt Berlin (Haus für Poesie) comme faisant partie des trois meilleurs débuts en poésie. 
Océan facile 4 on retrouve en effet ces vers de Mandelstam paraphrasés et répartis sur plusieurs lignes :
$[\ldots]$ eines tages wiederholt sich alles $[\ldots]$ zeit pendelt zurueck
$[\ldots]$
bis wir wieder erkennen:
$[\ldots]$ kostbar ist uns nur
der augenblick

des erkennens $[\ldots](\mathrm{CW}, 5)$

Cette double affirmation d'un temps cyclique et d'un temps de la (re)connaissance peut servir de biais pour entrer et cheminer dans ces poèmes d'Anne Seidel qui, au-delà de leur diversité formelle, présentent une forte cohérence sémantique et métaphorique obtenue par un réseau de répétitions de termes et de motifs, de parallélismes et de contrastes. La reprise du poème Tristia soulève une première question : qu'est-ce qui, dans cette totalité qui se répète, fait plus précisément retour dans le présent de sorte à susciter la reconnaissance (" wieder erkennen »), mais aussi la connaissance (« erkennen ») 5 ? Et selon quelle modalité ?

Le titre, singulier, Chlebnikov weint, la référence explicite au recueil de Mandelstam (« (tristia-)//versehrt», CW, 15) mais aussi à Constantin Batiouchkov, poète préromantique dont Mandelstam avait lu la traduction de l'élégie de Tibulle, et qui, à l'instar de Hölderlin, a sombré dans une " geistige Umnachtung $»^{6}$, montrent que ce retour se fait sur le mode élégiaque. Si Anne Seidel fait ostensiblement signe vers cette tradition générique (comme par exemple dans la constatation « von der traurigkeit unsrer kurzen saetze ende oktober bleibt eine erinnerung », $C W, 43)$, elle souligne fortement la dimension dialectique de la posture élégiaque. L'exergue qui ouvre le recueil le dit explicitement : « there is no better everything than loss if we have time ». Cette formule est extraite d'un poème de l'auteur américain Peter Gizzi (né en 1959) intitulé « Beginning with a phrase from Simone Weil » ${ }^{7}$. Dans ce poème,

4. - Cette expression, en français dans l'original allemand, est inspirée du titre d'un collage du poète et artiste surréaliste japonais Yamanaka Tiroux, Il y a un océan facile (1937).

5. - Le verbe russe « узнавать » signifie « reconnaître »; par le dédoublement/ redoublement « erkennen/wieder erkennen », Anne Seidel souligne la dimension de la connaissance. Je remercie Katia Bouchoueva et Aurélien Galateau qui m’ont aidé à lire les citations en russe présentes dans Chlebnikov weint.

6. - $C W, 35:$ « batjuškov, bei dir wird es frueher dunkel ». Né en 1787, Constantin Batiouchkov combine dans son œuvre la passion classique pour l'Antiquité romaine et le subjectivisme préromantique. Engagé volontaire dans les guerres napoléoniennes, auteur de poésies patriotiques et de traductions de la littérature italienne (Pétrarque, l'Arioste), il sombre dans la folie en 1822 et meurt en 1855.

7. - Peter Gizzi, Some Values of Landscape \& Weather, Wesleyan, Middletown, CT, 2003, p. 96. 
Peter Gizzi fait jouer, par une série de variations et de permutations d'une phrase de Simone Weil (« Tu ne pourrais être née à une meilleure époque que celle-ci où on a tout perdu $\rangle^{8}$ ), la dialectique du temps et de son abolition, de la continuité et de la discontinuité, du souvenir et de l'oubli, de la perte et du gain. "Beginning with a phrase from Simone Weil » commence par adapter la citation de la philosophe " there is no better time than present when we have lost everything " - pour progresser jusqu'à la question finale et sa réponse assertorique qui renvoie à l'ouvrage de Simone Weil, La Pesanteur et la Grâce : « Is there no better present than loss ? A grace opening to air. No better time than present », en passant par le paradoxe qu'Anne Seidel a retenu en ouverture de son recueil : " there is no better everything than loss if we have time. " Si le recueil semble donc d'emblée placé, du fait de l'intertexte de Peter Gizzi, sous le signe de la valorisation de l'instant présent, le deuxième exergue permet de comprendre qu'il ne s'agit pas pour Anne Seidel de revendiquer une tabula rasa qui ferait fi du passé. Cet exergue est extrait d'un poème de jeunesse de Guennadi Aïgui, dédié à Jiri Wolker, un poète tchèque lié au groupe d'avant-garde Devétsil, disparu à l'âge de 24 ans. L'exergue dit « что сказание все продолжается », c'est-à-dire : « que la légende se poursuit ». Il est question dans ce poème de gares à l'intérieur desquelles des moines concentrés écrivent en silence " que la légende se poursuit »9. La mise en exergue de la discontinuité temporelle et de la perte, garantes de l'instant présent chez Gizzi (et Simone Weil), est complétée par l'affirmation de la continuité d'une tradition qu'il faut lire (legenda) et écrire (à l'instar des moines-scribes).

Les différentes références et les noms que l'on trouve dans Chlebnikov weint ne constituent pas un simple name-dropping, un recours à des auctoritates chargées, selon l'étymologie, de garantir et d'augmenter la portée du discours poétique (d'ailleurs Mandelstam, dont l'ombre, avec celle de Walter Benjamin ou de Paul Celan, plane sur le recueil ${ }^{10}$, n'est pas nommé expressément). Il ne s'agit pas non plus de dresser aux poètes évoqués dans le recueil un monument mémoriel ni de les imiter, la référence à Khlebnikov dans le titre du recueil ne doit pas faire penser que l'écriture d'Anne Seidel emprunte au « zaoum » de Khlebnikov, langue «transrationnelle » créée selon le principe du déplacement (« svdig »), par dérivations étymologiques, glossolalie et

8. - Simone Weil, La Pesanteur et la grâce, Paris, Plon, 1988, p. 270.

9. - Poème original consultable en ligne à la page 366 de l'adresse : gennadiy20aygi-otmechennaya-20zima.pdf (consulté le 12/02/2019).

10. - Outre Khlebnikov, Aïgui, Gizzi et Batiouchkov sont aussi nommés Godard, Montale, Shakespeare, Komitas et Zajc. 
emprunts à diverses langues, si ce n'est à la marge ${ }^{11}$. Ces noms délimitent un champ de réflexions qui tournent autour du rapport au passé et à la résurgence de la poésie et de la culture. Quand « on a le temps », c'est-à-dire quand on possède la durée, on a le présent qui est l'instant où l'on fait accéder l'objet perdu (l'absence) au statut de présence. On pense à la conception mandelstamienne du poème comme instrument qui retourne la terre noire du temps, pour faire remonter à la surface du présent les traces de ce qui n'est plus, mais qui est capable aussi d'atteindre, par-delà le passé, des strates de temps « pur » :

La poésie est l'araire qui affouille le temps pour faire émerger les couches profondes, son tchernoziom. Mais l'humanité, à certaines époques où le quotidien ne l'assouvit plus, où elle languit de couches plus profondes, est, comme le laboureur, assoiffée des terres vierges du temps ${ }^{12}$.

Le cadre de cette réflexion sur le temps est donné par des notations issues d'un voyage réel ou imaginaire d'Anne Seidel dans l'espace russe et plus généralement slave : espaces essentiellement hivernaux, constitués de paysages de glace, d'étendues enneigées traversées de fleuves, mais aussi espaces littéraires et psychiques qui, tout autant que les contrées géographiques, portent les traces de l'Histoire, qu'elle soit proche ou lointaine. De poème en poème se dessine une cartographie qui relie la Biélorussie (Minsk) à la Volga (Saratov, abrégé en Sarat, $C W, 10-11)$, Saint-Pétersbourg et les rives de la Neva aux îles Solovki dans la Mer Blanche. Par la combinaison de fines notations de perceptions et de changements d'échelle abrupts, Anne Seidel arrive, dans l'espace restreint de poèmes généralement brefs, à suggérer l'immensité du territoire russe et de l'Europe orientale :

das schwarze ufer der erde hebt sich vor den beleuchteten wolken (hand des abends) tropfen zeichnend und geraeusche von sand : die kaelte der raender osteuropas besteht aus gluehenden wassertropfen an ungewaschenen fenstern - ihrem licht $(C W, 45)$

Ou encore:

11. - La musicalité des poèmes d'Anne Seidel repose sur des procédés somme toute classiques d'allitération, d'assonance, de permutation, comme par exemple : " schlacht am rande der iris, riss und schlacht, blauschlacht und firnis » $(C W, 19)$, « schwan und schwarz» $(C W, 20)$, on notera toutefois le néologisme « irrlichterlirende erinnerungen $\gg(C W, 34)$.

12. - Ossip Mandelstam, Euvres Complètes I, op.cit., p. 322. 


\section{zug durch belarus}

klirren silbern loeffel (in den teeglaesern der reisenden) hinein in die erinnerung an das novgoroder kirchenspiel, gibt es (den unbeirrbaren) weg des sich entziehenden (sich mir entziehender verzweifelter griff in das federkissen). hier: schwarze schienen halbverdeckt, das ueberholende ueberholt uns nicht, hat es nie. wir sind versunken mit dem letzten stein (klang des regens auf dem blechernen zugdach). ( $\mathrm{CW}, 49)$

Ces tableaux toutefois, qui, par leur réduction à des combinaisons de surfaces blanches et de signes noirs, peuvent évoquer des peintures suprématistes de Kasimir Malevitch, ne sont pas que des esquisses miniatures extraites d'un carnet de voyage. Ils font se télescoper des images du passé et du présent sous forme de constellations figuratives que l'on pourrait décrire avec ce que Walter Benjamin appelle dans son Livre des Passages « l'image dialectique ». L'historien, tel que l'imagine Benjamin, ne cherche pas à proposer une reconstruction du processus diachronique qui aurait mené progressivement du passé au présent, ni à s'approcher, à partir des connaissances du présent, de la réalité supposée du passé :

Il ne faut pas dire que le passé éclaire le présent ou que le présent éclaire le passé. Une image, au contraire, est ce en quoi l'Autrefois rencontre le Maintenant dans un éclair pour former une constellation. En d'autres termes, l'image est la dialectique à l'arrêt. Car, tandis que la relation du présent au passé est purement temporelle, la relation de l'Autrefois avec le Maintenant est dialectique : ce n'est pas quelque chose qui se déroule, mais une image saccadée. Seules les images dialectiques sont des images authentiques (c'est-à-dire non archaïques); et l'endroit où on les rencontre est le langage de nature figurative $\mathrm{e}^{13}$.

L'image dialectique est la rencontre violente du passé et du présent dans une image ouverte, polysémique qui demande à être interprétée. Ce moment fulgurant où la constellation se forme est comparable à l'instant du réveil où le dormeur sort du rêve, où la conscience se

13. - Walter Benjamin, Paris, Capitale du XIX $X^{e}$ siècle. Le livre des passages, trad. Jean Lacoste, Paris, Cerf, 1989, p. 478-479 (« Nicht so ist es, daß das Vergangene sein Licht auf das Gegenwärtige oder das Gegenwärtige sein Licht auf das Vergangene wirft, sondern Bild ist dasjenige, worin das Gewesene mit dem Jetzt blitzhaft zu einer Konstellation zusammentritt. Mit anderen Worten: Bild ist die Dialektik im Stillstand. Denn während die Beziehung der Gegenwart zur Vergangenheit eine rein zeitliche, kontinuierliche ist, ist die des Gewesnen zum Jetzt dialektisch: ist nicht Verlauf sondern Bild, sprunghaft. Nur dialektische Bilder sind echte (d.h.: nicht archaische) Bilder; und der Ort, an dem man sie antrifft, ist die Sprache. »W. Benjamin, Gesammelte Werke, Vol. V/ 1, sous la dir. de H. Schweppenhäuser et R. Tiedemann, Frankfurt a. M., Suhrkamp, 1991, p. 576-577. 
reconfigure. C'est précisément ce que peut la poésie par ses ruptures, ses procédés de montage, ses superpositions : «traeume sind von historischer bedeutung / und fragment », est-il dit dans le cycle « rueckseite des regens » $(C W, 47)$. Elle permet alors cet instant de la reconnaissance qui est aussi connaissance.

\section{unruhe der geschichte}

die welt war stumm, wir fuehlten uns verlassen.

als sie die weiße milch in den see goss,

schnitt der ss-mann glatt mit der hand durch die luft

\section{the dead are not around}

in diese passage: abend

in diese passage: schnee $(C W, 59)^{14}$

Ce poème, dont Anne Seidel explique en note qu'il a été inspiré par le film de Claude Lanzmann Shoah ${ }^{15}$, part d'une perspective « historique » (par la présence du terme « geschichte » et l'utilisation du prétérit) pour déboucher sur une citation en anglais (empruntée au film ?) au présent et un dernier vers dépourvu de verbe qui réunit, dans son indécision spécifique, les deux niveaux temporels. Le poème « unruhe der geschichte » associe la déréliction passée des victimes, le geste du SS dont on devine la signification meurtrière, et l'absence des morts dans le présent de l'écriture. La scène esquissée au centre du poème correspond dans son caractère énigmatique mais concret, à cette incarnation du souvenir qui, pour Lanzmann, permet d'éviter le « savoir mort »: " L'incarnation, c'est précisément ce que réalise l'œuvre d'art et c'est la raison pour laquelle, sans pourtant les dévaluer, elle est supérieure à toutes les autres formes de transmission, est la transmission même. $»^{16}$.

La présence discrète mais insistante du terme « passage » dans le recueil n'est pas qu'un hommage à Walter Benjamin ${ }^{17}$. Anne Seidel a vraisemblablement en tête la polysémie du terme : un passage désigne

14. — Le demi-vers « in diese passage : schnee » sert également de titre au dernier cycle du recueil.

15. - CW, 61 : « unruhe der geschichte/ Nach Shoah von Claude Lanzmann ».

16. - Claude Lanzmann, "Le rôle de l'art dans la transmission de la Shoah », in Ministère de l'éducation nationale, Mémoire et histoire de la Shoah à l'école, Paris, CNDP, p. 19-20, ici p. 20.

17. - Anne Seidel fait par exemple allusion à la gravure de Grandville Le pont des planètes que Benjamin commente dans le Passagenwerk. Sa définition de l'histoire maritime comme histoire de naufrages a des résonances éminemment benjaminiennes : "História trágico-marítima. Tragische Seegeschichte. Maritime Geschichte als Geschichte untergehender Schiffe » $(\mathrm{CW}, 43)$. 
un lieu de déambulation ou de transit, par exemple les galeries parisiennes analysées par Benjamin, ou un détroit maritime (de nos jours le passage du nord-ouest dans la mer des Tchouktches entre l'Alaska et la Sibérie est sillonné par un navire brise-glace russe appelé... « Kapitan Khlebnikov »), ou encore un segment que l'on parcourt dans un texte ; il désigne aussi le changement d'un état à un autre, le devenir, l'écoulement du temps. Le terme inscrit de la sorte le mouvement du temps dans l'espace, la disparition dans l'apparition, la trace sur la feuille (sur la neige) et son effacement, le silence dans la parole. Ce qui revient dans les poèmes d'Anne Seidel, ce n'est pas seulement le temps passé, c'est le temps en tant qu'il passe, c'est le mouvement qui fait retour et s'immobilise, se cristallise : « in diese passage : abend/in diese passage : schnee».

C'est pourquoi le recueil Chlebnikov weint est porté par la figure de la répétition mais aussi et surtout de la variation, tant au niveau de la macrostructure qu'à celui de la microstructure, ce qui est le propre d'une écriture cyclique. Il est articulé autour d'un grand cycle des « absences », comptant treize poèmes (sur quarante que totalise le recueil), intitulés chacun «Abwesenheiten » et numérotés avec des chiffres romains. Ce cycle des absences est divisé en deux volets qui encadrent un cycle central composé de six poèmes, intitulés chacun Hygiene der Angst, eux aussi numérotés avec des chiffres romains. Ces derniers poèmes sont tous construits sur le même modèle : ils comptent deux strophes, chacune de trois vers, la seconde répétant en grande partie des expressions de la première strophe, mais introduisant aussi des variations essentielles, notamment dans l'usage des temps. Cette construction binaire permet le télescopage du présent et du passé sous une forme qui s'apparente au collage. Les différences qui viennent troubler la quasi-identité des deux strophes attirent l'attention sur l'écart, sur l'altérité dans le retour du même, et constituent ce moment de la reconnaissance. Soit le quatrième poème du cycle :

\section{HYGIENE DER ANGST IV}

schwarze spitzen, weiße linien, russland, so hilflos zieht

stille ein, die namen getraenkt, ende der waelder,

es fehlte immer eine hand, versunken im pelz

schwarze spitzen, weiße linien, da warst du, so hilflos zog 
stille in dich ein, in namen und waelder,

es fehlte immer eine hand, versunken im schnee, solovki $(\mathrm{CW}, 28)$

Le poème évoque en deux brèves strophes la Russie et un de ses paysages spécifiques, le seuil où les forêts s'arrêtent et où commence un espace soumis à un hiver rigoureux, où les noms des hommes et des lieux sont imprégnés, - autant abreuvés que noyés -, de silence. Mais ce qui frappe à la lecture de ce poème, - que l'on pourrait rebaptiser à la façon d'un tableau : « Paysage de neige avec main absente »-, c'est le travail de subtile différenciation qui confronte et mélange présent et passé, voyage dans l'espace et dans le temps, présence d'une main en tant qu'elle est absente, enfouie dans la chaleur d'une pelisse et/ou dans le froid de la neige : « es fehlte immer eine hand, versunken im pelz/.../ es fehlte immer eine hand, versunken im schnee $»$. Le poème se termine par l'évocation d'un de ces noms imprégnés de silence : « solovki ». Nom chargé d'épaisseur temporelle et historique qui désigne l'archipel des Solovki ou Solotevsk dans la Mer Blanche, dont le monastère, devenu depuis le $\mathrm{XVI}^{\mathrm{e}}$ siècle un refuge pour les opposants au pouvoir tsariste, a été transformé à l'époque stalinienne en prison, puis en camp de travail forcé. L'évocation d'une main " absente » c'est-à-dire invisible parce qu'enfouie dans une pelisse, et d'une main absente parce que recouverte de neige, prend alors un sens particulier du fait même de la mention de « solovki », lieu de mémoire et métonymie du Goulag.

C'est le même principe qui est à l'œuvre dans le poème qui ouvre le cycle Hygiene der Angst :

\section{HYGIENE DER ANGST I}

statue, die den platz sezierte, hygiene der angst, eine kaelte, die schneesaum, geronnenes erdoel und einen melancholischen hund verzeichnet, leuchtendes andenken, logik des rauchgeruestes

statue, die den platz seziert, hygiene der angst, kaelte - stand: ein schweres rieseln durchschreitend, negantia, aufstuende ein melancholischer hund, vielleicht $(C W, 25)$

Ici aussi, Anne Seidel donne à voir un paysage, urbain cette fois, sous la forme d'une image qui juxtapose et superpose le passé et le présent : une statue (de style soviétique ?) qui, en disséquant la place (qu'il faut donc imaginer comme morte), pratique « cette hygiène de la peur » conférant son titre au cycle. Loin de tout pittoresque touristique, 
ce poème, au moyen d'une structuration qui superpose un chiasme (ABBA : passé/présent-présent/passé) et un entrecroisement (ABAC : « sezierte/verzeichnet/seziert/stand ») propose une image dialectique placée sous le signe de la mélancolie, mais aussi d'une ouverture " utopique ». Si, dans la première strophe, la neige, le pétrole gelé et le chien mélancolique semblent pétrifiés dans le froid, la deuxième strophe est beaucoup plus dynamique et ouvre sur l'optatif « aufstuende vielleicht $»^{18}$. Par l'image du chien mélancolique qui se dresserait, est envisagé un possible dépassement de la pétrification grâce au travail du négatif suggéré par l'utilisation du terme latin negantia, la négation. La mélancolie évoquée ici n'est pas l'acedia dénoncée par l'Église au moyen âge parce que mettant le moine dans un état dépressif qui le rend sensible aux sollicitations du Malin, c'est une mélancolie qui, par-delà la répétition du même (« statue, die den platz sezierte.../ statue, die den platz seziert ») semble peut-être en passe d'être surmontée.

Le poème est forme visible donnée à la présence absente qui fait retour : d'où l'utilisation de la forme cyclique qui non seulement structure l'ensemble du recueil mais figure aussi la répétition et ses variations. La répétition ne joue pas que sur des unités longues comme le cycle ou, à l'intérieur d'un poème, sur la strophe, on la retrouve au niveau inférieur du mot (« glomm-glomm », " schien schien schien » $(C W, 12)$, « schucht schucht schucht » $(C W, 30)$, « es zieht zieht zieht / er zieht zieht zieht/ sie zieht/gezogen/ gezogen : gezogen » $(C W, 32)$. Anne Seidel a également une prédilection pour les syntagmes comportant une répétition, « stufe um stufe » $(C W, 17)$, « nacht fuer nacht » $(C W$, $18)$, « stern um stern » $(C W, 21)$, mode de répétition qui se thématise lui-même : « wiederholung um wiederholung » $(C W, 37)$. Le doublon peut être fondu en un seul mot « silenceetsilence » $(C W, 22)$ ou venir scinder l'uni(ci)té affirmée : « raumeinziger-einziger» $(C W, 15)$. C'est la raison pour laquelle la différence est au cœur de l'identité, à l'instar du fameux fleuve d'Héraclite dont la permanence est garantie par le retour de l'altérité : « L'écoulement d'un ruisseau change d'une seconde à l'autre. Pour élargir Héraclite : "On est toujours dans un autre fleuve. Et alors que nous sommes déjà tournés vers autre chose, ce fleuve continue toujours à bruisser (autrement)" ${ }^{\prime 19}$.

18. - On notera l'utilisation récurrente de l'adverbe « vielleicht » chez Anne Seidel : $C W 10,15$ (2 fois), 27, 43.

19. - « Das Rinnen eines Baches unterscheidet sich von jeder Sekunde zur anderen. Um Heraklit zu erweitern: "Man ist schon immer in einem anderen Fluss. Und während wir uns schon etwas anderem zugewandt haben rauscht dieser Fluss immer noch (anders...)" » (Email à l'auteur du 27/02/2019). 
Le rapport au temps, à la disparition et à la résurgence, à l'unicité d'un surgissement et à la différence dans la répétition apparaît ainsi comme constitutif des poèmes d'Anne Seidel. Dans l'ordre métaphorique, la neige y a, comme chez Paul Celan, une valeur centrale de par sa polyvalence symbolique. Surface (im)maculée effaçant la trace autant qu'elle en constitue le support, facteur de cécité autant que de visibilité, analogon de la page blanche, elle a pour équivalent le silence qui est à la fois négation de la parole et sa condition d'apparition :

\section{Abwesenheiten VIII/silenceetsilence}

das auge voll schnee, der blick veraendert sich am abend auf der straße, beruehrt den boden an einigen stellen (hinterhof, sachlichkeit, alte zeitungen) vereiste tuerklinken, schneehaeufchen darauf, kalte wangen (leuchten zerbrochen, gestreutes krakelee) das licht, das ueber den eissee steigt, darunter die geraeusche, silenceetsilence, glaeserne schollen, felder, tiefste finsternis, schneise aus eis, verlorenes blatt in der mitte und alles ueberzaehlige wie tot. (schwimmende reusen) erdachte stoffe, in dir schneit es fabriken aus. $(\mathrm{CW}, 22)$

Ou encore :

die versunkene silence der worte

wie eine ackerfurche in wachsschnee mit zwei lichtern aus eisen die sickern $[\ldots](C W, 16)$

Si tout ce qui a disparu revient et, de ce fait, est susceptible d'être reconnu, le poème peut alors être qualifié de « forme de l'absence »: " gedicht (form der abwesenheit) », est-il dit dans " Abwesenheit III » $(C W, 17)$. Fonction qui est illustrée avec une concision remarquable dans un poème constitué d'une seule phrase répartie sur deux lignes :

\section{madame, sie sollten eigentlich nicht hier sein $(C W, 48)$}

Les caractères gras de cette phrase permettent de l'identifier, sur le modèle des textes qui l'entourent, à un titre de poème. Ce titre désigne, par l'apostrophe et le déictique « hier » une présence paradoxale : sous le titre s'étend la surface vide de la page, le titre n'est pas suivi d'un poème formulé, de sorte que le déictique " hier » acquiert un statut incertain. Il désigne un lieu qui est à la fois "dans le champ » et 
« hors-champ », où absence et présence sont indissociablement liées et se déterminent mutuellement. La présence de « madame » est dans le même mouvement de l'écriture, affirmée, regrettée et niée. Plus exactement, « madame » est absente dans l'affirmation de sa présence, présente dans la mise en scène de son absence.

De façon plus cryptée encore, Anne Seidel évoque le lien entre répétition et différence, présence et absence, profération et silence par la référence elliptique à une célèbre œuvre créée et performée par le compositeur américain Alvin Lucier. Le titre du premier poème de la série Abwesenheiten est en effet une citation : «Abwesenheiten $\mathrm{I} / \mathrm{i}$ am sitting in a room » fait allusion à l'œuvre de ce compositeur minimaliste précisément intitulée $i$ am sitting in a room (1970). Dans un premier temps, Alvin Lucier a enregistré en anglais le texte suivant qui décrit sa propre démarche :

Je suis assis dans une pièce différente de celle où vous vous trouvez maintenant. J'enregistre le son de ma voix et je vais repasser l'enregistrement dans la pièce encore et encore, jusqu'à ce que les fréquences provenant de la résonance de la pièce se renforcent elles-mêmes, de façon à ce que toute ressemblance avec ma parole, à part peut-être son rythme, soit détruite. Ce que vous entendrez alors seront les résonances naturelles de la pièce, articulées par ma voix. Je ne conçois cette activité pas tant comme la démonstration d'un phénomène physique, mais plus comme un moyen de lisser toutes les imperfections que ma voix pourrait avoir ${ }^{20}$.

Alvin Lucier diffuse ensuite l'enregistrement dans une pièce tout en le réenregistrant simultanément, et ce, plusieurs fois de suite, de sorte qu'au bout d'une douzaine d'enregistrements successifs, par le phénomène de feedback, l'enregistrement se trouve augmenté de ses propres résonances et harmoniques : la voix est devenue inintelligible, on n'entend plus qu'un son pur qui a été pour ainsi dire créé à partir du filtre que constitue l'espace et qui lentement se dissout dans le silence. La répétition, qui est au cœur du projet, ne crée pas du Même mais de la musique, l'insistance répétitive ne consolide pas le sens mais l'abolit, tout en transformant la parole en musique ${ }^{21}$. Anne Seidel voit dans ce

20. - « I am sitting in a room different from the one you are in now. I am recording the sound of my speaking voice and I am going to play it back into the room again and again until the resonant frequencies of the room reinforce themselves so that any semblance of my speech, with perhaps the exception of rhythm, is destroyed. What you will hear, then, are the natural resonant frequencies of the room articulated by speech. I regard this activity not so much as a demonstration of a physical fact, but more as a way to smooth out any irregularities my speech might have. » Consultable à l'adresse : http://www.multimedialab.be/blog/?p=2182 (consulté le 23/04/2019).

21. - On peut écouter cette performance à l'adresse : https://www.youtube. 
processus de surimpression de différents enregistrements le moyen de parvenir à un moment d'« existence pure », c'est-à-dire de poésie :

Lucier a enregistré un moment de pure existence et nous a intégrés (« different from the one you are in »). Retracer et analyser son travail ne signifie pas qu'on puisse saisir ce moment poétique, mais cela ne signifie pas non plus que ce soit impossible 22 .

Dans komitas und dane zajc in minsk $(C W, 55)$, Anne Seidel, s'inspirant d'un poème dans lequel l'écrivain slovène Dane Zajc travaille sur le nom mystérieux « Asskalla » en le faisant résonner et en le décomposant en ces différentes syllabes ( Asska/sska/lla/Ass/lla 1la lla/sska sska sska/lla lla Asskalla $»^{23}$ ), fait, à son tour, résonner le nom du compositeur et musicologue arménien Komitas, qui, suite aux persécutions dont il a été victime dans le cadre du génocide arménien, a été atteint d'aphasie et de troubles psychiatriques qui le poursuivront jusqu'a sa mort en exil en France, à l'hôpital psychiatrique de Villejuif : la décomposition du nom « Komitas » (« komitas... komita... kom i ta .. ») débouche sur le silence qui est suggéré par l'écho de la consonne finale : « So leise hallt dein stille gewordenes schweigen ass ass ass ».

Le recueil d'Anne Seidel cherche ainsi à se démarquer d'une écriture qui serait construction d'un mémorial, à contrecarrer la stratégie de l'oubli qui engloutit son objet par la mémoire :

Je faisais allusion à Musil qui disait en substance qu'avec les monuments commémoratifs autour du cou, on précipitait les individus dans l'oubli. Ce que l'anglais appelle « sensing », aide à contrecarrer «l'oubli qui se souvient ». L'individu qui avait en lui une vie précieuse indépendamment de son travail, se dresse devant son œuvre. C'est bien sûr quelque chose qu'on ne peut guère exprimer. En fait, ce qui m'importe, c'est une attitude. Le déplacement sublime modifie ma perception. De ce fait, un silence s'établit avant la parole. De ce fait, l'écoute est autre, la parole est autre. Et bien sûr, la lecture, elle aussi, s'en trouve modifiée. Le savoir se soumet à cette reconnaissance de l'expérience pure que j'exprime ${ }^{24}$.

com/watch? $\mathrm{v}=$ fAxHILK3Oyk (consulté le 03/02/2019). Alvin Lucier présente cette performance comme une façon de dépasser son bégaiement.

22. - « Lucier hat einen Moment reinen Daseins aufgenommen und uns einbezogen ('different from the one you are in now'). Sein Schaffen zu umreißen und dazu zu forschen bedeutet nicht diesen poetischen Moment fassen zu können, aber das heißt auch nicht, dass es unmöglich wäre. 》> (Email à l'auteur du 09/02/2019).

23. - Dane Zajc, Erdsprache, Auswahl und Übertragung aus dem Slowenischen von Fabjan Hafner, Klagenfurt/Celovec, Wieser Verlag, 1990, p. 55. On peut entendre Dane Zajc dire son poème à l'adresse https://www.youtube.com/watch? $v=J z b A t 7 s \_2 V M$ (consulté le 14/02/2019).

24. - « Ich spielte auf Musil an, der irgendwo etwas in der Art sagte wie: 
C'est pourquoi le recueil Chlebnikov weint est placé sous le signe de la dialectique du souvenir et de l'oubli : " Ich erinnere mich, dass ich mich an nichts erinnere, denn nichts/zu verstehen, ist die einzige moeglichkeit, etwas zu verstehen ", est-il dit en guise de programme au seuil du recueil $(C W, 9)$. Poétique qui place Anne Seidel dans la lignée de Mandelstam et de Celan, lesquels ne conçoivent la parole poétique et la remémoration que comme un surgissement qui fait la part de l'obscurité et de l'oubli, de la clarté et de l'hermétisme. « Ne rien comprendre afin de mieux comprendre », c'est ici aussi faire jouer la dialectique de la perte et du gain. Dès la première lecture du recueil, on perçoit nettement l'intérêt d'Anne Seidel pour la force poétique de certains mots étrangers qui doivent être perçus et ressentis en tant que tels, indépendamment de considérations sur la compréhension ou l'incompréhension qu'ils pourront susciter : on trouve dans Chlebnikov weint des mots et des expressions en français (« silence », « à contre-cœur », « le pont des planètes », « une autre présence »), en tchèque (« poduska » " pustoty ») ${ }^{25}$, en espagnol (" História trágico-maritima »), en russe (« les », « sneg ») ${ }^{26}$, en anglais (« the dead are not around »), et, comme je l'ai déjà relevé, des exergues en russe et en écriture cyrillique ${ }^{27}$. La présence de citations en caractères cyrilliques soulève la question du statut de ces blocs erratiques pour les lecteurs qui, n'ayant pas forcément été mis en contact avec la culture russe comme ce fut le cas à l'époque de la RDA, ignorent et le russe et l'alphabet cyrillique. Ces blocs n'ont pas pour vocation à être sautés par un lecteur non russophone, ils peuvent, selon Anne Seidel, susciter, en tant que signes, un autre type de « lecture » par-delà leur signification :

Je pars du principe que les caractères d'une écriture ont, en tant que symboles, une influence sur notre conscience, mais aussi sur la pensée concrète et les émotions. Posséder une langue/ une écriture ou ne pas les posséder, chacune de ces possibilités a son charme : pouvoir percevoir une écriture dans son esthétique sans sa signification ou voir

dass man mit Denkmälern um den Hals die Menschen ins Vergessen stürze. Was im Englischen ,Sensing' genannt wird, hilft gegen das ,erinnernde Vergessen'. Vor seinem Werk steht der Mensch, der ganz unabhängig von seinem Schaffen wertvolles Leben in sich barg. Das ist natürlich etwas, das sich kaum konkret äußern lässt. Es geht mir eigentlich eher um eine Haltung. Die sublime Verschiebung ändert meine Perzeption. Da ist eine Stille vor dem Sprechen, die dadurch aufkommt. Das Schreiben ist dadurch ein anderes, das Sprechen ist dadurch ein anderes. Und natürlich verändert sich das Lesen. Das Wissen richtet sich diesem meinem Ausdruck der Anerkennung des reinen Daseins unter. » (Email à l'auteur du 09/02/2019).

25. - «Coussin, oreiller» et « vide, vacuité ».

26. — « Forêt» et « neige ».

27. - Le poème Sarat $I(C W, 10)$ compte aussi un exergue anonyme en caractères cyrilliques dont la signification est : "Qui a écrit le poème ? J'ai écrit le poème ». 
derrière les signes leur signification sont deux possibilités enrichissantes de la contemplation phénoménologique qui peuvent souvent, de façon complémentaire, se dérouler en même temps. Quelqu'un qui ne parle pas la langue est quand même « touché » par l'écriture. Il voit ce que le locuteur ne peut pas voir 28 .

Ce qui nous ramène à la fonction des noms propres dans ce recueil, en particulier celui de Khlebnikov. Le nom est ce qui de la personne demeure après sa disparition. Si on peut dire, avec Emmanuel Levinas, que le visage constitue « l'épiphanie » du sujet individuel, le nom, une fois la personne morte, renvoie simultanément à son épiphanie et à sa disparition. Il n'est pas indispensable que le lecteur connaisse l'œuvre du poète russe, ni les raisons exactes pour lesquelles il «pleure », de même qu'il peut « lire » les caractères cyrilliques sans les comprendre. Vélimir Khlebnikov avait de nombreuses raisons de " pleurer », notamment à la fin de sa vie, pendant les années de guerre civile, au cours de son errance entre Ukraine, Perse et Azerbaïdjan. Dans un de ses tout derniers textes, il écrit : « ...alors j'ai couvert mes yeux avec mes ailes et suis mort pour la deuxième fois, après avoir longuement sangloté : ce monde - qu'il est plein d'affliction! $»^{29}$ Mais Anne Seidel ne cherche pas à faire un portrait biographique ou historique, à reconstituer ce qu'a pu être la vie misérable de Khlebnikov qui échappe à la conscription en se faisant admettre dans un hôpital psychiatrique et qui, revenu dans le nord de la Russie, meurt du typhus en 1922 près de Novgorod. Comme Thomas Kling, mais dans une langue poétique très différente, elle opère un montage qui dit la rencontre de l'Autrefois et du Maintenant avec tout ce que la (re)connaissance que cela produit peut contenir d'effrayant :

\begin{tabular}{lc}
\multicolumn{2}{c}{-zwei } \\
$\begin{array}{ll}\text { abgebrochene graphitspitzen } & \text { auf altem papier } \\
\text { die uns immer noch } & \text { erschrecken koennen }\end{array}(C W, 13)$
\end{tabular}

28. — «Die Schriftzeichen haben als Symbole, wie ich annehme, einen ästhetischen Einfluss auf unser Bewusstsein und auch auf das konkrete Denken und die Emotionen. Beides, eine Sprache/Schrift zu beherrschen und eine Sprache/Schrift nicht zu beherrschen, hat seine Schönheit: eine Schrift in ihrer Ästhetik ohne ihre Bedeutung wahrnehmen zu können und hinter den Zeichen ihre Bedeutung zu sehen sind zwei gewinnbringende Möglichkeiten der phänomenologischen Betrachtung, die oft ganz unterbewusst gleichzeitig ablaufen können. Jemand der die Sprache nicht spricht wird dennoch von der Schrift „getroffen“. Er sieht, was der Sprecher nicht sehen kann. » (Email à l'auteur du 09/01/2019). Plus généralement, les citations en cyrillique, ainsi que l'abandon définitif de la graphie « $\ddot{a} »$, , « $\ddot{~} »$, , ü » au profit de « ae », « oe » et « ue », participent de cet effet d'« ostranenie », de « défamiliarisation » ou « désautomatisation », dont parle Viktor Chklovski à propos de la fonction de l'art, et dont se réclame Anne Seidel.

29. - Vélimir Khlebnikov, Euvres. 1919-1922, traduit du russe, préfacé et annoté par Yvan Mignot, Lagrasse, Verdier, 2017, p. 974. 